\title{
Frequency, Causes, and Outcomes of Return Visits to the Emergency Department Within 72 Hours: A Retrospective Observational Study
}

This article was published in the following Dove Press journal: Journal of Multidisciplinary Healthcare

\author{
Mohammed Alshahrani (iD) \\ Faisal Katbi iD ${ }^{2}$ \\ Yazeed Bahamdan (iD ${ }^{3}$ \\ Ahrar Alsaihati ${ }^{4}$ \\ Aisha Alsubaie (iD ${ }^{5}$ \\ Dana Althawadi ${ }^{5}$ \\ Laila Perlas-Asonto id ${ }^{6}$ \\ 'Departments of Emergency and Critical \\ Care, King Fahd Hospital of the \\ University, Imam Abdulrahman Bin Faisal \\ University, Al-Khobar, 31952, Kingdom of \\ Saudi Arabia; ${ }^{2}$ Department of Emergency \\ Medicine, King Fahd Hospital of the \\ University, Imam Abdulrahman Bin Faisal \\ University, Dammam, Kingdom of Saudi \\ Arabia; ${ }^{3}$ Department of General \\ Pediatrics, Children's Hospital- King Saud \\ Medical City, Riyadh, Saudi Arabia; \\ ${ }^{4}$ Department of Dermatology, King Fahd \\ Hospital of the University, Imam \\ Abdulrahman Bin Faisal University, \\ Dammam, Kingdom of Saudi Arabia; \\ ${ }^{5}$ Department of Emergency, King Hamad \\ University Hospital, Busaiteen, Kingdom \\ of Bahrain; ${ }^{6}$ Department of Emergency \\ and Critical Care Medicine, King Fahd \\ Hospital of the University, Imam \\ Abdulrahman Bin Faisal University, \\ Dammam, Kingdom of Saudi Arabia
}

Correspondence: Mohammed Alshahrani Department of Emergency and Critical Care Medicine, King Fahd Hospital of the University, Imam Abdulrahman Bin Faisal University, P.O. Box 40236, Al-Khobar

31952, Kingdom of Saudi Arabia

Tel +966-556-966663

Email msshahrani@iau.edu.sa
Background: Emergency departments (EDs) serve as an accessible gateway to healthcare system wherein numerous patients consider it a prime choice for medical complaints. Frequency of ED revisits, causes, and its burden are necessary to assess quality of care provided to patients and identify factors that leads to revisit.

Patients and Methods: Electronic and printed medical records of all patients who revisited ED from January to May 2016 within 72 hours of initial visit were reviewed. Patients' cause of revisit were classified to three categories: patient-, physician- and system-related factors. Common complaints that require revisits were also collected. Descriptive analysis was performed and categorical variables were represented by the frequency; percentages and continuous variables were presented as median, and range if data did not follow normal distribution.

Results: Of the 79,279 patients who visited ED during the study period, $1.3 \%$ (1000) patients revisited within 72 hours; $51.3 \%(n=513)$ were males, with a mean age of 31.5 years ( $\mathrm{SD}=17.7$ years) where majority $(57.1 \%)$ had no comorbidity recorded. The most attributed factors for revisit were as follows: patient-related causes 635 patients $(63.5 \%)$, physician-related factors 167 patients $(16.7 \%)$, and system-related factors 42 patients $(4.2 \%)$; $15.6 \%$ were found not related to the initial visit. Recurrence of the same complaint was the highest among patient-related factors $(80.5 \%)$, inadequate management and no improvement of symptoms in $71.3 \%$ among the physician-related factors. The most common ED revisit complaint was fever $29.1 \%(n=291)$. Outcomes of the revisit were mainly patient discharge $96.7 \%(\mathrm{n}=967)$, admission $1.2 \%(\mathrm{n}=12)$ and death in $0.2 \%(\mathrm{n}=2)$.

Conclusion: Recurrence of the same complaint with no symptoms improvement and suboptimal management of physicians contributed to most of the ED revisits within 72 hours. Encouraging physicians to provide clear instructions in educating patients on discharge regarding disease progression and its red flags as to when a return to ED, might help in reducing revisit rate.

Keywords: emergency department, revisit, factors

\section{Introduction}

Emergency Department (ED) return visit is a known quality indicator for patient care and safety in EDs worldwide. Patients returning to the ED within a short period from initial presentation contribute to overcrowding ${ }^{1-4}$ which leads to delayed treatment, patient displeasure, waste of ED resources and increased costs of health care. ${ }^{1,2,5,6}$

Patients who return to the ED within 72 hours of discharge are often recognized to have received inappropriate treatment or evaluation. ${ }^{7}$ Return visit rates of more than $5 \%$ may reflect poor quality of care, and rates less than $1 \%$ indicate undue risk 
aversion. $^{8}$ ED return visits within 72-hours can be categorized into three groups, namely: illness-related, physician-related and patient-related ED returns. ${ }^{9}$ In illnessrelated ED returns, the patient receives appropriate medical care, but disease progression causes the ED return visit. In physician-related ED returns, the patient returns due to the physician's misconduct. Unexpected ED return visits were found to be associated with medical errors in treatment, follow-up care, prognosis, and information. ${ }^{10}$ In patient-related ED return visits there is no clear evidence of lapses in medical care and return visits are mainly patient-initiated. However, it is difficult to differentiate between the disease course nature, sub-optimal therapy, patient anxiety, and medical error misbehavior as the cause of the ED return visit. ${ }^{11}$ Other factors such as increasing age of the patient, illness severity, and inexperienced or junior physicians were found to be associated with ED return visits. ${ }^{10,12-16}$

One study conducted at a hospital in Canada on ED return visits showed that of the 9935 ED visits, 289 (2.9\%) were within 72 hours. Most unscheduled ED return visits were classified as low-acuity (45.3\%), and in most of them $(88.6 \%)$, patients were treated in the ED and discharged home. ${ }^{17}$ Another study by Lerman and Kobernick reported that of the 64,336 ED visits during the period of the study, 255 returned to ED within 72 hours. Of them, 83 (32.5\%) ED return visits were avoidable by better education of patients or medical care on the initial visit. ${ }^{18}$

With rising ED return visit rates, there has been an increasing need to address the problem of overcrowding. ${ }^{19,20}$ Analyzing factors associated with ED return visit will help in decreasing avoidable ED return visits and reducing the financial health care burdens. The global data propose that a rate of early ED return visits of approximately $3 \%$ is an acceptable estimation of the average international return visit rate. ${ }^{21}$ However, there is a debate in the literature about the acceptable rate of ED return rates and the factors associated with ED revisits. The time span of early ED return visits may identify the rate of early return visits to the ED. Longer time spans increases the rate of early ED return visits and include patients who are either frequent attendees, with chronic disease, or unrelated attendance. Most of the studies used a 72-hour period between initial and following visits. ${ }^{21}$ Furthermore, some studies included all early ED return visits with both related and unrelated diagnoses, ${ }^{11,22,23}$ and other studies included only early ED return visits with related diagnoses. ${ }^{13}$
In this study, we aimed to identify the rate and characteristics of return visit at our ED within 72 hours from the initial visit and to determine clinical and demographic factors that include patient age, acuity of triage level, and presence of comorbidities, clinician education level and diagnoses. Likewise, we also hope to determine the underlying factors associated with unscheduled ED return visits and thereby help the application of strategies to address overcrowding, communication improvement and promote patient care.

\section{Patients and Methods}

\section{Study Design and Setting}

A retrospective observational study where electronic and printed medical record review of all patients who revisited the emergency department of King Fahad University Hospital (KFHU)- Eastern province of Saudi Arabia, within 72 hours of their initial emergency visit was conducted. KFHU has an ED annual visit of approximately 200,000 patients per year.

Description and metrics of the department showed patient to staff ratio is $3: 1$ in the observation unit and $1: 1$ in resuscitation and trauma area. 46 physicians (24 Saudi and 22 non- Saudi). Time to provider of 26.6 minutes and average length of stay in the department is 2.6 hours.

We chose 72 hours as a revisit cutoff in this study as comparable to previous studies made with similar outcomes and this is only for unscheduled revisits.

\section{Study Duration}

The study included patients who revisited the emergency department within the period from January 2016 to May 2016. This five-month period was chosen to overcome the seasonal variation that might influence the revisit rate.

\section{Study Variables}

In this study, revisit was defined as the patient visiting the ED after 72 hours from their initial ED visit. Predetermined factors chosen with an agreement by investigating team prior to the study start were used where the revisit reasons were categorized to physician-related, patient-related and systemrelated factors. Physician-related included suboptimal management defined as misdiagnosis and complications of treatment; patient-related factors included recurrence of same complaints, no improvement of symptoms, and complication of a disease; whereas system-related factors 
included follow-up, called back for abnormal investigations, unscheduled refill of medication and for admission.

\section{Source of Data}

Authors identified patients included in the study within a five-month period using a generated electronic report from hospital patient- visit database. A previously designed and pre-piloted tested data form was used to collect patient data from the electronic and printed medical records by co-investigators who are ED residents and interns (FK, AA, SA, DA, YB) that were trained by the principal investigator (PI). In case of any disparity or clarity, the PI was contacted to resolve the disagreement.

The data form is attached as a supporting information.

\section{Eligibility Criteria}

We included all patients who revisited the ED within 72 hours of their initial emergency visit within the study period. All patients were included irrespective of their age or gender. We excluded patients who came after 72 hours and patients who were seen in other EDs, as no previous visit data were available.

\section{Statistical Analysis}

All categorical variables were represented by the frequencies and percentages. Data normality was tested by the Kolmogorov-Smirnov test. Variables presented as mean and standard deviation (SD) or median with inter-quartile range (IQR) as appropriate.

Continuous variables were presented as a median and range if the data did not follow the normal distribution.
Table I Demographic Characteristics of the Study Participants

\begin{tabular}{|c|c|}
\hline Variables & $\begin{array}{l}\text { Descriptive Statistics } \\
(n=1000)\end{array}$ \\
\hline \multicolumn{2}{|l|}{ Gender } \\
\hline Male & $513(51.3 \%)$ \\
\hline Female & 487 (48.7\%) \\
\hline \multicolumn{2}{|l|}{ Age in Years } \\
\hline Mean (SD) & $31.5(17.7)$ \\
\hline Median (Range) & $30(I-90)$ \\
\hline \multicolumn{2}{|l|}{ Age category } \\
\hline Pediatrics & $249(24.9 \%)$ \\
\hline Adult & 751 (75.1\%) \\
\hline \multicolumn{2}{|l|}{ Top 4 diagnosis } \\
\hline Upper respiratory tract infection & 79 (7.9\%) \\
\hline Bronchial asthma & $31(3.1 \%)$ \\
\hline Gastritis & $25(2.5 \%)$ \\
\hline Sickle cell disease & $22(2.2 \%)$ \\
\hline
\end{tabular}

\section{Results}

79,279 patients visited ED during the study period, among them, 1000 patients had to revisit within 72 hours which represents $1.3 \%$. Of those $513(51.3 \%)$ were males, and $48.7 \%(n=487)$ were females with a mean age of 31.5 years $(\mathrm{SD}=17.7$ years). Most of patients $(57.1 \%)$ had no comorbidity, followed by hypertension (11\%), and bronchial asthma $10.5 \%$. The demographic characteristics of the study participants are shown in Table 1 while their past medical history is shown in Figure 1.

\section{Characteristics of the Initial Visits and the Revisits}

Initial ED assessment of subjects were carried out by residents in training $63.5 \%(\mathrm{n}=635)$, followed by $13.5 \%$

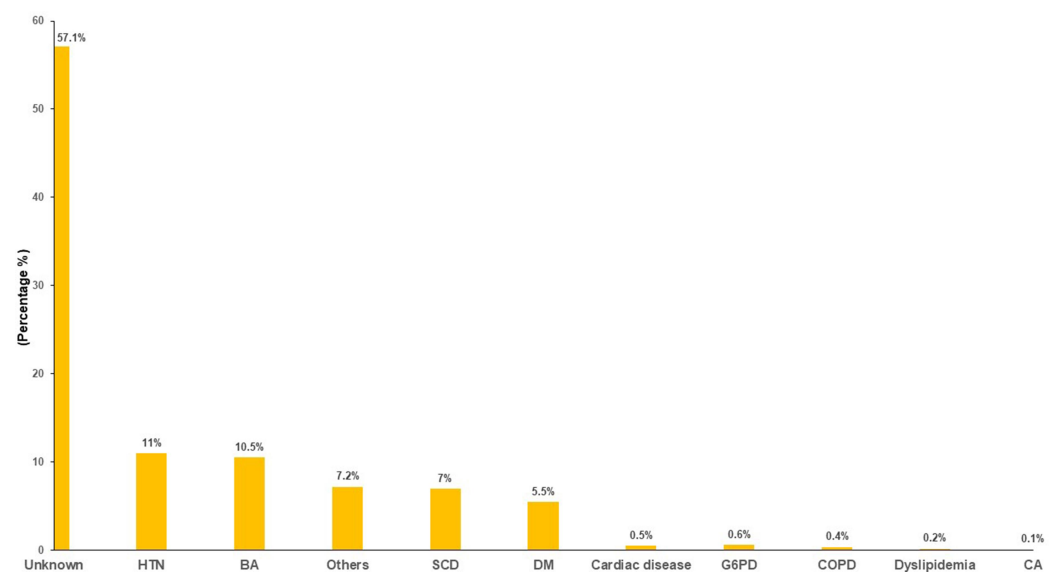

Figure I The past medical history of subjects. 
$(n=135)$ assessed by the general practitioners, $11.9 \%$ ( $n=119)$ by specialists (board certified EM physicians), Consultants (licensed after 3 years of certification) assessed $0.5 \%(n=5)$ of subjects, and in $10.6 \%(n=106)$ the assessor was unclear during the chart review and that was not statistically significant between both groups $\mathrm{p}$ value $=0.569$.

The order of treatment was organized based on the degree of urgency with three different triage levels (III, IV, V), the triage level III accounts for $2.7 \%(n=27)$, followed by triage level IV with $62.4 \%(n=624)$ and the triage level $\mathrm{V}$ with $34.9 \%(\mathrm{n}=349) \mathrm{p}$ value $=0.711$. The outcome of treatment was assessed with a discharge rate of $95.7 \%$ ( $n=957)$, followed by discharged against medical advice (DAMA) $0.9 \%$, lack of availability of bed $0.4 \%$, admission of $0.3 \%$.

Most of the revisit patients were of triage category IV with $59.8 \%(n=594)$. The outcomes of the revisit to the ED were mainly discharge of $96.7 \%(n=967)$, admission of $1.2 \%(n=12)$ and death in $0.2 \%(n=2)$. The characteristics of the initial visit and the revisits are shown in Table 2.

Table 2 Characteristics of the Initial Visit and the Revisits

\begin{tabular}{|l|l|l|l|}
\hline & Initial Visit & Revisit & P value \\
\hline $\begin{array}{l}\text { Assessor } \\
\text { Resident }\end{array}$ & $635(63.5 \%)$ & $610(61 \%)$ & 0.569 \\
Staff physician & $135(13.5 \%)$ & $132(13.2 \%)$ & \\
Specialist & $119(11.9 \%)$ & $137(13.7 \%)$ & \\
Consultant & $5(0.5 \%)$ & $3(0.3 \%)$ & \\
Unknown & $106(10.6 \%)$ & $118(11.8 \%)$ & \\
\hline Triage level & & & 0.711 \\
I III & & $1(0.1 \%)$ & \\
IV & $27(2.7 \%)$ & $33(3.3 \%)$ & \\
V & $624(62.4 \%)$ & $594(59.8 \%)$ & \\
\hline Length of stay & $349(34.9 \%)$ & $365(36.7 \%)$ & \\
Mean(SD) & $1.57(2.3)$ & $1.57(2.1)$ & 0.031 \\
Median (Range) & $1.0(6$ minutes - & $1.0(4$ minutes - & \\
& 24 hours) & 19 hours) & \\
\hline Final Outcome & & & 0.601 \\
Discharged & $957(95.7 \%)$ & $967(96.7 \%)$ & \\
DAMA* & $9(0.9 \%)$ & & \\
No bed available & $4(0.4 \%)$ & $12(1.2 \%)$ & \\
Admitted & 0 & $2(0.2 \%)$ & \\
Expired & 0 & $19(1.2 \%)$ & \\
Unknown & $27(2.7 \%)$ & & \\
\hline
\end{tabular}

Note: *Discharge against medical advice.

\section{Causes and Complaints of the Revisits}

Patient-related causes for ED revisits were the most reported attributed factors seen in 635 patients $(63.5 \%)$, followed by physician-related factors in 167 patients $(16.7 \%)$ and the least were system-related factors seen in 42 patients $(4.2 \%)$. The frequencies of physician, patient, and system-related causes of ED visits are shown in Figures 2-4, respectively.

The recurrence of the same complaint was the highest among the patient-related factors $(80.5 \%)$ and suboptimal management and no improvement of symptoms in $71.3 \%$ among the physician-related factors. The most common ED revisit complaints were fever 29.1\% $(n=291)$, then abdominal pain and vomiting in $12.4 \%(n=124)$, followed by body and back pain in $9.7 \%(\mathrm{n}=97)$, headache in $9.5 \%$ $(n=95)$, cough in $8.5 \%(n=85)$, trauma in $6 \%(n=60)$, dizziness in $3 \%(n=30)$, shortness of breath in $2 \%(n=20)$ and others $19.8 \%(n=198)$. The complaints of initial visits and revisits are shown in Table 3. Furthermore, those factors can be overlapped due to different reasons however, the final determination of which factor was the main cause of the revisit was based on the investigators agreement. Agreement of $100 \%$ was observed with regards to inter-rater reliability between physicians who evaluated charts.

\section{Discussion}

In this study 79,279 patients visited the ED during the study period. We found that the overall proportion of ED return visits within three days was $1.6 \%$. This was consistent with multiple studies published on ED return visits rates using a determination of return visits of within 72 hours after the initial ED visits, with results ranging between $1.3 \%$ to $7.5 \% .^{11,13,22-24}$ Most of these studies included early ED returns using both related and unrelated diagnoses. One retrospective study in Florida and Nebraska published a rate of all ED return visits as up to $7.5 \%{ }^{23}$ Another study, conducted at a medical center in northern Taiwan, reported that the monthly ED return visit rates ranged from $1.3 \%$ to $2.4 \% .{ }^{24}$ Another study at a regional hospital in central Taiwan reported higher monthly rates ranging from $2.85 \%$ to $6.25 \%{ }^{11}$ Keith et $\mathrm{al}^{22}$ reviewed the charts of all ED patients in a US-based hospital; they found that $455(3.4 \%)$ of the 13,261 patients returned to the ED within 72 hours. Another study conducted in Singapore reported a return rate of $2 \%$ excluding the returns for unrelated diagnoses. ${ }^{13}$ Our study showed that both genders have almost equal chances of early ED 


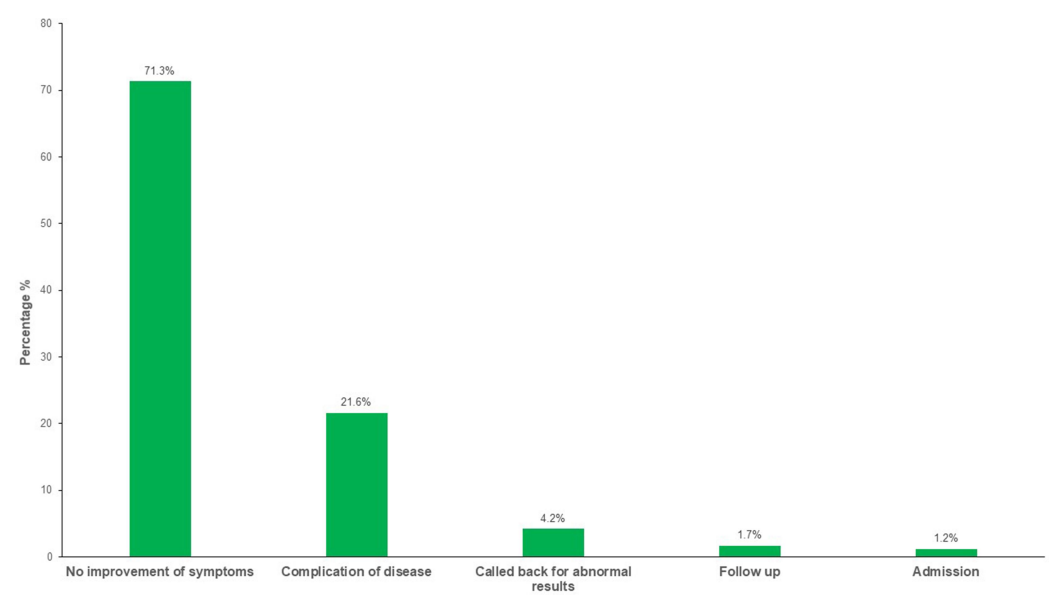

Figure 2 The frequency of physician-related causes of ED revisits.

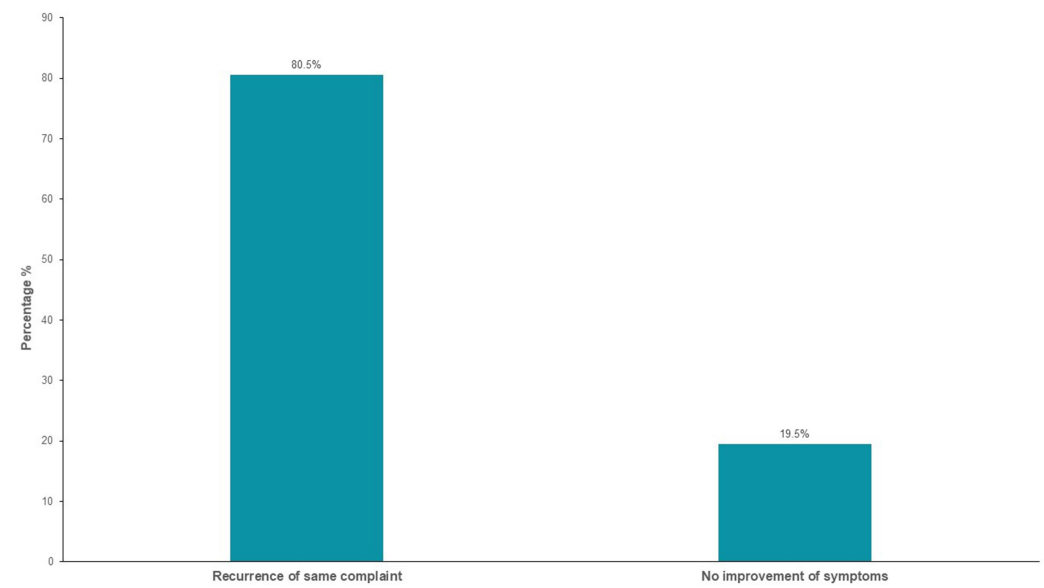

Figure 3 The frequency of patient-related causes of ED revisits.

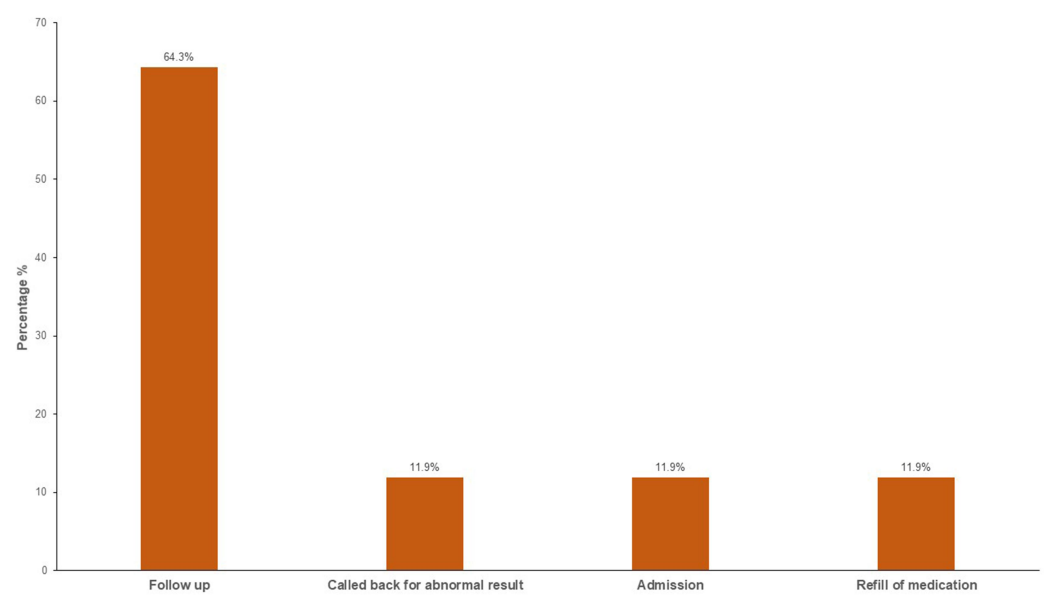

Figure 4 The frequency of system-related causes of ED revisit.

return visits. In contrast with other studies, where another study in Taiwan on the ecology of medical care published that a higher proportion of men (87.6/1000 vs $81.0 / 1000)$ received emergency services in 2005 compared with women. ${ }^{25}$ Multiple studies found that women were more health conscious and that men usually delay seeking 
Table 3 Complaints of the Initial Visit and Revisits

\begin{tabular}{|l|l|l|l|}
\hline & Initial Visit & Revisit & P value \\
\hline Vomiting & $40(4 \%)$ & $21(2.1 \%)$ & 0.109 \\
Shortness of breath & $33(3.3 \%)$ & $20(2.0 \%)$ & 0.447 \\
Trauma & $41(4.1 \%)$ & $60(6.0 \%)$ & 0.269 \\
Headache & $36(3.6 \%)$ & $95(9.5 \%)$ & $0.003^{*}$ \\
Fever & $245(24.5 \%)$ & $291(29.1 \%)$ & 0.341 \\
Dizziness & $63(6.3 \%)$ & $30(3.0 \%)$ & 0.801 \\
Back pain & $86(8.6 \%)$ & $41(4.1 \%)$ & $0.027^{*}$ \\
Abdominal Pain & $95(9.5 \%)$ & $103(10.3 \%)$ & 0.567 \\
Cough & $49(3.9 \%)$ & $85(8.5 \%)$ & $0.002^{*}$ \\
Body pain & $103(10.3 \%)$ & $56(5.6 \%)$ & $<0.001 *$ \\
Others & $209(20.9 \%)$ & $198(19.8 \%)$ & 0.847 \\
\hline
\end{tabular}

Notes: *Level of significance.

medical help until later stage of their diseases. ${ }^{26,27}$ Moreover, women may have a lower rate of employment, giving them more time to visit doctors. ${ }^{28}$

Our study found a mean age of 31.5 years for ED patients who had return visits, where previous study in comparison found that people aged more than 64 years composed the majority of patients with early ED return visits. ${ }^{21}$ Most of the revisit patients were of triage category IV with 59.8\% $(n=594)$ using Canadian Triage and Acuity Scale (CTAS). Resident physicians initially managed $63.5 \%(n=635)$ of the revisit patients because the highacuity patients are generally more unwell and usually seen by senior physicians (specialists and consultants), while the lower acuity patients (category IV) are seen by junior physicians (residents) that may be associated to more ED return visits. These findings were consistent with the previous studies which reported that junior or inexperienced clinicians were associated with ED return visits. ${ }^{12,13}$ The outcomes of the revisit to the ED were mainly discharged since most of the revisits were category IV in their initial visit. Majority of them had no comorbidities, which is expected due to mean young age in our study compared to other studies, followed by hypertension and bronchial asthma. In our study, the most common ED revisits complaints were fever, then abdominal pain and vomiting, followed by back pain. Recurrence of the same complaint was the highest among the patient-related factors and suboptimal management and no improvement of symptoms in among the physician-related factors. Compared with other studies they found factors associated with higher risk for early return visits ED diagnoses were heart problems, abdominal pain or viral infections. ${ }^{29}$ Other studies have identified the three greatest risk factors in the 72-hour re- attendees group; they found that heart problems, viral infection, and abdominal pain were the most significant risk factors. ${ }^{9,11,30,31}$

A literature review on early ED return visits showed that there were variations in the probable causes and that they remain not clearly defined. ${ }^{1,7,9,11,18,22,30,32,33}$ In the future, we aim to look beyond the index visit and investigate if there was a considerable difference in diagnosis and disposition status between the index and ED return visit. Also, it would be essential to examine admission outcomes of the ED return in the subgroup of 72-hour who were admitted on their return visit, particularly complication rates, as research published showed that patients who are admitted on the ED return visit have poor outcomes. ${ }^{34} \mathrm{We}$ conclude that this study is one of the first to analyze ED return visits in the Middle East population. Our rate of return visit might indicate the quality of care given at the ED. These findings would help in conducting further research as well as directing future strategies to decrease markedly avoidable ED return visit.

We believe that the strength of our study is being conducted in one of the largest and busiest emergency departments in the Eastern Province of Saudi Arabia, with annual visits between 180,000-200,000 visits/year, which gives a good sample to calculate the frequency of revisit rate among population on this region. However, a higher rate of discharge in our department was found and is mostly related to many people with low acuity complaints in this region prefer to go to the ED which is considered easier access than primary health care centers. Nevertheless, as this retrospective study relied on accurate data collection and documentation, interpretation may have been limited by information or selection bias. This study was conducted over a 5 month period however, this cannot guarantee overcoming seasonal variation which can lead to different presentations which might affect the results. During extraction of patient information from files and electronic records, we were unable to obtain some detailed information about some of the patients; we did not have information on some patient characteristics, such as compliance with treatments, socioeconomic status, level of education, and confidence in their primary care, which are associated with ED return visits and might confound the results. And finally, this study was also limited by being a single center experience and no other centers data can be compared. Also there was no evidence that the non-reattenders did not seek other EDs in the region for medical treatment. 


\section{Conclusion}

In this study, recurrence of the same complaint, no improvement of symptoms and sub-optimal management by the physician, as well as being initially assessed by a resident physician contributed to most of the ED returns within 72 hours. Even though there is no direct evidence of this, accentuating and encouraging physicians to giving patients clear instructions and education on the disease process upon discharge and identifying red flags for when to return to the ED might help in decreasing the revisits rate.

\section{Abbreviation}

ED, emergency department; CTAS, Canadian Triage Acuity Scale.

\section{Data Sharing Statement}

All data produced and analyzed in this study are included in this manuscript as presented in Tables 1-3 and Figures $1-4$.

All data requests should be addressed to the corresponding author for consideration. Access to anonymised data may be granted following review. All source records including electronic data are stored in secured systems in accordance with institutional policies and regulations. Data including any electronic transmission of data are only identified by the study coded number.

The study results will be released to the participating physicians, patients and the general medical community on publication.

\section{Ethics Approval and Consent to Participate}

No patients were involved either in the design, recruitment and conduct of this study nor in the development of outcome measures. We will publish the results of the study in lay language for patient interest groups. This study, including data acquisition was approved by the Ethics Committee of Imam Abdulrahman Bin Faisal University Ethics Board approval no. IRB-2015-01-136. All patient data was de-identified and patient consent acquisition was waived by our ethics board due to the retrospective nature of this study.

The manuscript does not contain any individual person's data and is compliant based on Declaration of Helsinki. Standing Committee for Research Ethics on Living Creatures (SCRELC) of the Imam Abdulrahman bin
Faisal University and the authors have no objection in granting and assigning the Journal of Multidisciplinary Healthcare unrestricted right to reproduce, publish, and distribute this manuscript in all forms.

\section{Acknowledgments}

We wish to acknowledge the King Fahad Hospital of the University administrators and staff for providing much needed assistance throughout the course of this research. We thank our colleagues from the ED who provided insight and expertise that greatly assisted the research and for their comments on an earlier version of the manuscript.

This article is accessible in the book of abstracts which was presented in Research Forum ACEP17 in October 29-31, 2017; Walter E. Washington Convention Center, Washington DC.

\section{Author Contributions}

The corresponding author had full access to all data presented in this study and the final responsibility of deciding to submit the study for publication. All authors made significant contributions to conceptualization, design, acquisition, analysis and interpretation of data. Likewise, all authors took part in drafting and critically revising the article for essential intellectual content; agreed to submit to the current journal; gave final approval of the version to be published; and agreed to be accountable for all aspects of the work.

\section{Funding}

Authors declare that they receive no financial support from any commercial organization or company.

\section{Disclosure}

The authors report no conflicts of interest for this work.

\section{References}

1. Goldman RD, Ong M, Macpherson A. Unscheduled return visits to the pediatric emergency department - one-year experience. Pediatr Emerg Care. 2006;22:545-549. doi:10.1097/01.pec.0000230553.01917.05

2. Goldman RD, Kapoor A, Mehta S. Children admitted to the hospital after returning to the emergency department within 72 hours. Pediatr Emerg Care. 2011;27:808-811. doi:10.1109/CPRE.2018.8349823

3. Cho CS, Shapiro DJ, Cabana MD, et al. A national depiction of children with return visits to the emergency department within 72 hours, 2001-2007. Pediatr Emerg Care. 2012;28:606-610. doi:10.10 97/PEC.0b013e31825cf7cf

4. Health D of London. A\&E Clinical Quality Indicators: Implementation Guidance. Department of Health London; 2010. 
5. Berry A, Brousseau D, Brotanek JM, et al. Why do parents bring children to the emergency department for nonurgent conditions? A qualitative study. Ambul Pediatr. 2008;8:360-367. doi:10.1016/j. ambp.2008.07.001

6. Hoot NR, Aronsky D. Systematic review of emergency department crowding: causes, effects, and solutions. Ann Emerg Med. 2008;52:126-136.e1. doi:10.1016/j.annemergmed.2008.03.014

7. Benbassat J, Taragin M. Hospital readmissions as a measure of quality of health care: advantages and limitations. Arch Intern Med. 2000;160:1074. doi:10.1001/archinte.160.8.1074

8. Heyworth J. Emergency medicine-quality indicators: the United Kingdom perspective. Acad Emerg Med. 2011;18:1239-1241. doi:10.1111/j.1553-2712.2011.01223.x

9. Ng C, Chung C. An analysis of unscheduled return visits to the accident and emergency department of a general public hospital. Hong Kong J Emerg Med. 2003;10:153-161. doi:10.1177/102490790301000304

10. Nuñez S, Hexdall A, Aguirre-Jaime A. Unscheduled returns to the emergency department: an outcome of medical errors? Qual Saf Health Care. 2006. doi:10.1136/qshc.2005.016618

11. Wu CL, Wang FT, Chiang YC, et al. Unplanned emergency department revisits within 72 hours to a secondary teaching referral hospital in Taiwan. J Emerg Med. 2010;38:512-517. doi:10.1016/j.jemermed. 2008.03.039

12. Ross MA, Hemphill RR, Abramson J, et al. The recidivism characteristics of an emergency department observation unit. Ann Emerg Med. 2010. doi:10.1016/j.annemergmed.2010.02.012

13. Kuan WS, Mahadevan M. Emergency unscheduled returns: can we do better? Singapore Med J. 2009;50:1068-1071.

14. Abualenain J, Frohna WJ, Smith M, et al. The prevalence of quality issues and adverse outcomes among 72-hour return admissions in the emergency department. J Emerg Med. 2013;45:281-288. doi:10.10 16/j.jemermed.2012.11.012

15. Martin-Gill C, Reiser RC. Risk factors for 72-hour admission to the ED. Am J Emerg Med. 2004. doi:10.1016/j.ajem.2004.07.023

16. Mccusker J, Cardin S, Bellavance F, Belzile É. Return to the emergency department among elders: patterns and predictors. Acad Emerg Med. 2000;7:249-259. doi:10.1111/j.1553-2712.2000.tb01070.x

17. Foran A, Wuerth-Sarvis B, Milne WK. Canada. Mar Pollut Bull. 1994;28:345. doi:10.1016/0025-326X(94)90268-2

18. Lerman B, Kobernick MS. Return visits to the emergency department. J Emerg Med. 1987;5:359-362. doi:10.1016/07364679(87)90138-7

19. Boyle A, Beniuk K, Higginson I, Atkinson P. Emergency department crowding: time for interventions and policy evaluations. Emerg Med Int. 2012;2012:1-8. doi:10.1155/2012/838610

20. Cowan RM, Trzeciak S. Clinical review: emergency department overcrowding and the potential impact on the critically ill. Crit Care. 2005;9:291. doi:10.1186/cc2981
21. Trivedy CR, Cooke MW. Unscheduled return visits (URV) in adults to the emergency department (ED): a rapid evidence assessment policy review. Emerg Med J. 2015;32:324-329. doi:10.1136/ emermed-2013-202719

22. Keith KD, Bocka JJ, Kobernick MS, et al. Emergency department revisits. Ann Emerg Med. 1989;18:964-968. doi:10.1016/S01960644(89)80461-5

23. Rising KL, Victor TW, Hollander JE, Carr BG, Griffey RT. Patient returns to the emergency department: the time-to-return curve. Acad Emerg Med. 2014;21:864-871. doi:10.1111/acem.12442

24. Liaw SJ, Bullard MJ, Hu PM, et al. Rates and causes of emergency department revisits within 72 hours. J Formos Med Assoc. 1999.

25. Shao -C-C, Chang C-P, Chou L-F, et al. The ecology of medical care in Taiwan. J Chin Med Assoc. 2011;74:408-412. doi:10.1016/j. jcma.2011.08.005

26. Pinkhasov RM, Wong J, Kashanian J, et al. Are men shortchanged on health? Perspective on health care utilization and health risk behavior in men and women in the United States. Int $J$ Clin Pract. 2010;64:475-487. doi:10.1111/j.1742-1241.2009.02290.x

27. Banks I. No man's land: men, illness, and the NHS. BMJ. 2001;323:1058-1060. doi:10.1136/bmj.323.7320.1058

28. Directorate-General of Budget. Accounting and statistics, executive Yuan, ROC (Taiwan); 2012. Available from: http://www.stat.gov.tw/ public/Attachment/081918204771.doc. Accessed December 7, 2020.

29. Chan AHS, Ho SF, Fook-Chong SMC, et al. Characteristics of patients who made a return visit within 72 hours to the emergency department of a Singapore tertiary hospital. Singapore Med J. 2016. doi:10.11622/smedj.2016104

30. Pham JC, Kirsch TD, Hill PM, et al. Seventy-two-hour returns may not be a good indicator of safety in the emergency department: a national study. Acad Emerg Med. 2011;18:390-397. doi:10.1111/ j.1553-2712.2011.01042.x

31. Pierce JM, Kellerman AL, Oster C. "Bounces": an analysis of shortterm return visits to a public hospital emergency department. Ann Emerg Med. 1990;19:752-757. doi:10.1016/S0196-0644(05)81698-1

32. O’Loughlin K, Hacking KA, Simmons N, et al. Paediatric unplanned reattendance rate: A\&E clinical quality indicators. Arch Dis Child. 2013;98:211-213. doi:10.1136/archdischild-2012-302836

33. Hasan M. Readmission of patients to hospital: still ill defined and poorly understood. Int J Qual Heal Care. 2001;13:177-179. doi:10.1093/intqhe/13.3.177

34. Shy BD, Shapiro JS, Shearer PL, et al. A conceptual framework for improved analyses of 72-hour return cases. Am J Emerg Med. 2015;33:104-107. doi:10.1016/j.ajem.2014.08.005

\section{Publish your work in this journal}

The Journal of Multidisciplinary Healthcare is an international, peerreviewed open-access journal that aims to represent and publish research in healthcare areas delivered by practitioners of different disciplines. This includes studies and reviews conducted by multidisciplinary teams as well as research which evaluates the results or conduct of such teams or healthcare processes in general. The journal covers a very wide range of areas and welcomes submissions from practitioners at all levels, from all over the world. The manuscript management system is completely online and includes a very quick and fair peer-review system. Visit http://www.dovepress.com/testimonials. php to read real quotes from published authors. 